\title{
A MATHEMATICAL MODEL OF COMPETITION FOR TWO ESSENTIAL RESOURCES IN THE UNSTIRRED CHEMOSTAT*
}

\author{
JIANHUA WU ${ }^{\dagger}$, HUA NIE,,$^{\dagger}$, AND GAIL S. K. WOLKOWICZ
}

\begin{abstract}
A mathematical model of competition between two species for two growth-limiting, essential (complementary) resources in the unstirred chemostat is considered. The existence of a positive steady-state solution and some of its properties are established analytically. Techniques include the maximum principle, the fixed point index, and numerical simulations. The simulations also seem to indicate that there are regions in parameter space for which a globally stable positive equilibrium occurs and that there are other regions for which the model admits bistability and even multiple positive equilibria.
\end{abstract}

Key words. chemostat, essential or complementary resources, steady-state solution, fixed point index, numerical simulation

AMS subject classifications. 35K55, 35J65, 92A17

DOI. $10.1137 / \mathrm{S} 0036139903423285$

1. Introduction. An apparatus called the chemostat, used for the continuous culture of microorganisms, has played an important role in ecology. It has been thought of as a lake in a laboratory. See $[9,25,29]$ for a description of the apparatus and the general theory.

In the basic set up, the culture vessel is assumed to be well stirred. One or more populations of microorganisms grow and/or compete exploitatively for a single, nonreproducing, growth-limiting nutrient that is supplied at a constant rate. The contents of the culture vessel are removed at the same constant rate as the medium containing the nutrient is supplied, and thus the volume of the culture vessel remains constant. Species-specific parameters can be measured one species at a time, and based on these parameters the theory predicts the qualitative outcome in advance of actual competition. In particular, the theory predicts that the species with the lowest break-even concentration excludes all other competitors (see $[6,14,29])$. Experiments confirmed this prediction in the case of auxotrophic bacterial strains competing for limiting tryptophan [11].

Mathematical analysis of chemostat models involving two limiting resources under the assumption that the culture vessel is well stirred can be found, for example, in $[2,3,7,13,12,17,18,19,28]$. When more than one resource is limiting, it is necessary to consider how these resources promote growth. At one extreme are resources that are sources of different essential substances that must be taken together, because each substance fulfills different physiological needs with respect to growth, for example, a

${ }^{*}$ Received by the editors February 22, 2003; accepted for publication (in revised form) February 6, 2004; published electronically September 24, 2004. This work was supported in part by the Natural Science Foundation of China, the Excellent Young Teachers Program of the Ministry of Education of China, the Foundation for University Key Teacher of the Ministry of Education of China, the Scholarship Foundation of CSC, the Institute for Mathematics and its Applications with funds provided by the NSF, and by the Natural Sciences and Engineering Research Council of Canada.

http://www.siam.org/journals/siap/65-1/42328.html

$\dagger$ Department of Mathematics, Shaanxi Normal University, Xi'an, Shaanxi 710062, People's Republic of China (wjhua@snnu.edu.cn).

${ }^{\ddagger}$ Department of Mathematics and Statistics, McMaster University, Hamilton, Ontario, Canada L8S 4K1 (wolkowic@mcmaster.ca). 
carbon source and a nitrogen source. Such resources are called complementary by Leon and Tumpson [17], Rapport [22], and Baltzis and Fredrickson [4]; essential by Tilman [28]; and heterologous by Harder and Dijkhuizen [12].

The model of exploitative competition for two essential resources in the wellstirred case is given by

$$
\begin{aligned}
& S_{t}=\left(S^{0}-S\right) D-\frac{1}{y_{s_{1}}} g_{1}(S, R) u-\frac{1}{y_{s_{2}}} g_{2}(S, R) v, \\
& R_{t}=\left(R^{0}-R\right) D-\frac{1}{y_{r_{1}}} g_{1}(S, R) u-\frac{1}{y_{r_{2}}} g_{2}(S, R) v, \\
& u_{t}=\left[-D+g_{1}(S, R)\right] u, \\
& v_{t}=\left[-D+g_{2}(S, R)\right] v .
\end{aligned}
$$

$S(t), R(t)$ denote the nutrient concentrations at time $t$, and $u(t)$ and $v(t)$ denote the biomass of each population in the culture vessel. $S^{0}>0$ and $R^{0}>0$ are constants that represent the input concentrations of nutrients $S$ and $R$, respectively, $D$ is the dilution rate, and $y_{s_{i}}$ and $y_{r_{i}}, i=1,2$, are the corresponding growth yield constants. The response functions are denoted $g_{i}(S, R)=\min \left(p_{i}(S), q_{i}(R)\right), i=1,2$, where $p_{i}(S)$ denotes the response function of the $i$ th population when only resource $S$ is limiting and $q_{i}(R)$ denotes the response function of the $i$ th population when only resource $R$ is limiting. We will consider the case that the Monod model for exploitative competition for one resource is generalized to the two essential resources case, i.e., $p_{i}(S)=\frac{m_{s_{i}} S}{K_{s_{i}}+S}$, $q_{i}(R)=\frac{m_{r_{i}} R}{K_{r_{i}}+R}, i=1,2$, where $m_{s_{i}}, m_{r_{i}}, K_{s_{i}}, K_{r_{i}}$, are positive constants.

In this paper, we study the unstirred chemostat and consider two species' competition for two, growth-limiting, nonreproducing essential resources. Motivated by the work on the unstirred chemostat in the case of one limiting resource (see $[5,8,15,16$, $20,23,24,25,26,30,31])$ and in the case of two limiting resources in [32], the model takes the form of the following reaction-diffusion equations:

$$
\begin{array}{ll}
S_{t}=d S_{x x}-\frac{1}{y_{s_{1}}} g_{1}(S, R) u-\frac{1}{y_{s_{2}}} g_{2}(S, R) v, & 0<x<1, t>0, \\
R_{t}=d R_{x x}-\frac{1}{y_{r_{1}}} g_{1}(S, R) u-\frac{1}{y_{r_{2}}} g_{2}(S, R) v, & 0<x<1, t>0, \\
u_{t}=d u_{x x}+g_{1}(S, R) u, & 0<x<1, t>0, \\
v_{t}=d v_{x x}+g_{2}(S, R) v, & 0<x<1, t>0,
\end{array}
$$

with boundary conditions

$$
\begin{array}{ll}
S_{x}(0, t)=-S^{0}, & R_{x}(0, t)=-R^{0}, u_{x}(0, t)=0, v_{x}(0, t)=0, \\
S_{x}(1, t)+\gamma S(1, t)=0, & R_{x}(1, t)+\gamma R(1, t)=0 \\
u_{x}(1, t)+\gamma u(1, t)=0, & v_{x}(1, t)+\gamma v(1, t)=0 .
\end{array}
$$

The boundary conditions are very intuitive. Readers may refer to $[5,16,26]$ for their derivation.

These equations can be simplified using the nondimensional variables and parameters defined as follows: $\bar{S}=\frac{S}{S^{0}}, \bar{R}=\frac{R}{R^{0}}, \alpha=\frac{S^{0} y_{s_{1}}}{R^{0} y_{r_{1}}}, \beta=\frac{R^{0} y_{r_{2}}}{S^{0} y_{s_{2}}}, \bar{g}_{i}(\bar{S}, \bar{R})=$ $\min \left(\frac{m_{s_{i}} \bar{S}}{\bar{K}_{s_{i}}+\bar{S}}, \frac{m_{r_{i}} \bar{R}}{\bar{K}_{r_{i}}+\bar{R}}\right), i=1,2, \bar{u}=\frac{u}{y_{s_{1}} S^{0}}, \bar{v}=\frac{v}{y_{r_{2}} R^{0}}$, where $\bar{K}_{s_{i}}=\frac{K_{s_{i}}}{S^{0}}, \bar{K}_{r_{i}}=\frac{K_{r_{i}}}{R^{0}}$, $i=1,2$. For more convenient notation, we drop the bars on the nondimensional 
variables and parameters, yielding the following model:

$$
\begin{array}{ll}
S_{t}=d S_{x x}-g_{1}(S, R) u-\beta g_{2}(S, R) v, & 0<x<1, t>0, \\
R_{t}=d R_{x x}-\alpha g_{1}(S, R) u-g_{2}(S, R) v, & 0<x<1, t>0, \\
u_{t}=d u_{x x}+g_{1}(S, R) u, & 0<x<1, t>0, \\
v_{t}=d v_{x x}+g_{2}(S, R) v, & 0<x<1, t>0,
\end{array}
$$

with boundary conditions

$$
\begin{aligned}
& S_{x}(0, t)=-1, \quad R_{x}(0, t)=-1, \quad u_{x}(0, t)=0, v_{x}(0, t)=0, \\
& S_{x}(1, t)+\gamma S(1, t)=0, \quad R_{x}(1, t)+\gamma R(1, t)=0, \\
& u_{x}(1, t)+\gamma u(1, t)=0, \quad v_{x}(1, t)+\gamma v(1, t)=0,
\end{aligned}
$$

and initial conditions

$$
\begin{aligned}
& S(x, 0)=S_{0}(x) \geq 0, R(x, 0)=R_{0}(x) \geq 0, u(x, 0)=u_{0}(x) \geq 0, v(x, 0)=v_{0}(x) \geq 0 . \\
& \text { Denote } \varphi_{1}=S+u+\beta v, \varphi_{2}=R+\alpha u+v, \text { where } \varphi_{i}, i=1,2, \text { is the solution of } \\
& \quad \quad 0<x<1, t>0, \\
& \varphi_{i t}=d \varphi_{i x x}, \\
& \varphi_{i x}(0, t)=-1, \quad \varphi_{i x}(1, t)+\gamma \varphi_{i}(1, t)=0, \\
& \varphi_{i}(x, 0)=\varphi_{i 0}(x) .
\end{aligned}
$$

Then $u$ and $v$ satisfy

$$
\begin{array}{ll}
u_{t}=d u_{x x}+u g_{1}\left(\varphi_{1}-u-\beta v, \varphi_{2}-\alpha u-v\right), & 0<x<1, t>0, \\
v_{t}=d v_{x x}+v g_{2}\left(\varphi_{1}-u-\beta v, \varphi_{2}-\alpha u-v\right), & 0<x<1, t>0, \\
u_{x}(0, t)=0, \quad u_{x}(1, t)+\gamma u(1, t)=0, & t>0, \\
v_{x}(0, t)=0, \quad v_{x}(1, t)+\gamma v(1, t)=0, & t>0 .
\end{array}
$$

This paper is devoted to determining the positive solution of this two-species model of exploitative competition for two essential resources in the unstirred chemostat. Since the reaction terms are Lipschitz continuous, but not $C^{1}$, many methods used to analyze elliptic systems do not apply. This makes the analysis more difficult. Some methods used to prove the existence of the positive equilibrium in the region $D=\left\{\left(\hat{\lambda}_{1}, \hat{\lambda}_{2}\right): \hat{\lambda}_{1}>1, \hat{\lambda}_{2}>1\right\}$ occupy a major portion of the paper, where $\hat{\lambda}_{i}, i=1,2$, is defined in the next section. The main result is established in Theorem 3 . The other related results are also obtained in section 2. Extensive numerical studies were run, and some conclusions are summarized in section 3 . The simulations convince us that much more complex dynamics can occur in region $D$.

The paper is organized as follows. In section 2 , the existence of a positive steadystate solution and some of its properties are established by using the maximum principle and fixed point index theory, which is closely related to bounding the principal eigenvalues of certain differential operators. Some results on extensive numerical studies are reported in section 3, complementing the mathematical results in section 2 , and a number of typical figures chosen from many simulations are also given in this section. 
2. The positive steady-state solution. First, we consider the steady state of system (1):

$$
\begin{array}{ll}
d S_{x x}-g_{1}(S, R) u-\beta g_{2}(S, R) v=0, & 0<x<1, \\
d R_{x x}-\alpha g_{1}(S, R) u-g_{2}(S, R) v=0, & 0<x<1, \\
d u_{x x}+g_{1}(S, R) u=0, & 0<x<1, \\
d v_{x x}+g_{2}(S, R) v=0, & 0<x<1,
\end{array}
$$

with boundary conditions

$$
\begin{aligned}
& S_{x}(0)=-1, \quad R_{x}(0)=-1, \quad u_{x}(0)=0, v_{x}(0)=0, \\
& S_{x}(1)+\gamma S(1)=0, \quad R_{x}(1)+\gamma R(1)=0, \quad u_{x}(1)+\gamma u(1)=0, \quad v_{x}(1)+\gamma v(1)=0 .
\end{aligned}
$$

It follows that $S+u+\beta v=z, R+\alpha u+v=z$, where $z=z(x)=\frac{1+\gamma}{\gamma}-x$. Then $u$ and $v$ satisfy

$$
\begin{array}{ll}
d u_{x x}+u g_{1}(z-u-\beta v, z-\alpha u-v)=0, & 0<x<1, \\
d v_{x x}+v g_{2}(z-u-\beta v, z-\alpha u-v)=0, & 0<x<1, \\
u_{x}(0)=0, & u_{x}(1)+\gamma u(1)=0, \\
v_{x}(0)=0, & v_{x}(1)+\gamma v(1)=0 .
\end{array}
$$

Let $\lambda_{i}$ be the principal eigenvalue and let $\phi_{i}(x)>0$ on $[0,1], i=1,2$, be the corresponding eigenfunction, normalized as $\max _{x \in[0,1]} \phi_{i}(x)=1$, of the following problem:

$$
d \phi_{i x x}+\lambda_{i} \phi_{i} g_{i}(z, z)=0, \quad 0<x<1, \quad \phi_{i x}(0)=0, \quad \phi_{i x}(1)+\gamma \phi_{i}(1)=0 .
$$

Let $U(x)$ be the solution of

$$
\begin{aligned}
& d U_{x x}+U g_{1}(z-U, z-\alpha U)=0, \quad 0<x<1, \\
& U_{x}(0)=0, \quad U_{x}(1)+\gamma U(1)=0,
\end{aligned}
$$

and let $U(x, t)$ be the solution of

$$
\begin{aligned}
& U_{t}=d U_{x x}+U g_{1}\left(\varphi_{1}-U, \varphi_{2}-\alpha U\right), \quad 0<x<1, t>0, \\
& U_{x}(0, t)=0, \quad U_{x}(1, t)+\gamma U(1, t)=0, \\
& U(x, 0)=U_{0}(x) \geq 0
\end{aligned}
$$

From Lemmas 2.2-2.4 and Theorem 2.5 in [32] we have the following lemma.

LEMma 1. If $\lambda_{1}<1$, then there exists a unique positive solution $U(x)$ of (5), satisfying $0<U<\min \left\{1, \frac{1}{\alpha}\right\} z$ on $[0,1]$. If $\lambda_{1} \geq 1$, the only nonnegative solution of (5) is $U=0$. Furthermore, $\lim _{t \rightarrow \infty} U(x, t)=U(x)$ if $\lambda_{1}<1$, and $\lim _{t \rightarrow \infty} U(x, t)=0$ if $\lambda_{1}>1$.

Remark 1. If $\lambda_{2}<1$, a similar result holds for $V(x)$, where $V(x)$ is the unique positive solution of

$$
\begin{aligned}
& d V_{x x}+V g_{2}(z-\beta V, z-V)=0, \quad 0<x<1, \\
& V_{x}(0)=0, \quad V_{x}(1)+\gamma V(1)=0 .
\end{aligned}
$$


Since we are only concerned with the nonnegative steady-state solutions of (3), there is no loss of generality if we redefine

$$
p_{i}(S)=\left\{\begin{array}{ll}
\frac{m_{s_{i}} S}{K_{s_{i}}+S}, & S \geq 0, \\
0, & S<0,
\end{array} \quad q_{i}(R)= \begin{cases}\frac{m_{r_{i}} R}{K_{r_{i}}+R}, & R \geq 0, \\
0, & R<0 .\end{cases}\right.
$$

Lemma 2. Suppose $(u, v)$ is the nonnegative solution of (3). Then (i) $u>0$ or $u \equiv 0$, and $v>0$ or $v \equiv 0$; (ii) $u+\beta v<z, \alpha u+v<z$; (iii) $u \leq U, v \leq V$. Moreover, $u<U$ or $u \equiv U$, and $v<V$ or $v \equiv V$.

Proof. (i) This part can be proved by the maximum principle, in the usual way, and the details are omitted here.

(ii) Define $w=u+\beta v-z$. Note that by (3) it follows that

$$
\begin{gathered}
d w_{x x}+u g_{1}(-w, z-\alpha u-v)+\beta v g_{2}(-w, z-\alpha u-v)=0, \\
w_{x}(0)=1 \text { and } w_{x}(1)+\gamma w(1)=0 .
\end{gathered}
$$

First we show that $w \leq 0$ on $[0,1]$. Suppose not. If $w(1)>0$, then $w_{x}(1)<0$. Therefore, there exists $a \in[0,1)$ so that for all $x \in(a, 1], w(x)>0$, and either $a=0$ or $w(a)=0$. But then for all $x \in[a, 1], w_{x x}=0$ and so $w_{x}(x)=w_{x}(1)<0$, i.e., $w(x)$ is decreasing there. Since $w_{x}(0)=1>0, a \neq 0$. But $a>0$ is also impossible since then $w(a)=0, w(x)$ is decreasing in $[a, 1]$, and $w(1)>0$. Therefore, $w(1) \leq 0$. Next, assume there exists $\bar{x} \in[0,1)$ with $w(\bar{x})>0$. Then there exist $\delta_{1} \geq 0$ and $\delta_{2}>0$ such that $w(x)>0$ for all $x \in\left(\bar{x}-\delta_{1}, \bar{x}+\delta_{2}\right) \subset(0,1), w\left(\bar{x}+\delta_{2}\right)=0$, and either $\bar{x}-\delta_{1}=0$ or $w\left(\bar{x}-\delta_{1}\right)=0$. But then for all $x \in\left[\bar{x}-\delta_{1}, \bar{x}+\delta_{2}\right], \quad w_{x x}(x)=0$ and so $w_{x}(x)$ is constant. Since $w\left(\bar{x}+\delta_{2}\right)=0$, it follows that $w_{x}\left(\bar{x}+\delta_{2}\right) \leq 0$, and so $w(x)$ is nonincreasing on $\left[\bar{x}-\delta_{1}, \bar{x}+\delta_{2}\right]$. Then $\bar{x}-\delta_{1} \neq 0$, since $w_{x}(0)=1$, and so $w\left(\bar{x}-\delta_{1}\right)=0$. Therefore, $w(x) \equiv 0$ on $\left[\bar{x}-\delta_{1}, \bar{x}+\delta_{2}\right]$, a contradiction. Hence, $u+\beta v \leq z$ on $[0,1]$. That $\alpha u+v \leq z$ follows similarly. It is easy to see that $u+\beta v \not \equiv z, \quad \alpha u+v \not \equiv z$; otherwise we have $d u_{x x}=0, d v_{x x}=0$, with the usual boundary condition, which gives $u \equiv 0, v \equiv 0$, a contradiction. Let $w_{1}=z-u-\beta v, w_{2}=z-\alpha u-v$. Then $w_{i} \geq 0, \not \equiv 0$, and $w_{1}$ satisfies

$$
\begin{aligned}
& -d w_{1 x x}+u g_{1}\left(w_{1}, w_{2}\right)+\beta v g_{2}\left(w_{1}, w_{2}\right)=0, \\
& w_{1 x}(0)=-1, \quad w_{1 x}(1)+\gamma w_{1}(1)=0,
\end{aligned}
$$

which leads to

$$
\begin{aligned}
& -d w_{1 x x}+w_{1}\left(\frac{m_{s_{1}} u}{K_{s_{1}}+w_{1}}+\frac{m_{s_{2}} \beta v}{K_{s_{2}}+w_{1}}\right) \geq 0 \\
& w_{1 x}(0)=-1, \quad w_{1 x}(1)+\gamma w_{1}(1)=0 .
\end{aligned}
$$

If $w_{1}\left(x_{0}\right)=0$ for some point $x_{0} \in[0,1]$, by applying the strong maximum principle (see [21]) we obtain a contradiction. Hence $w_{1}>0$ on $[0,1]$. The proof that $w_{2}>0$ on $[0,1]$ is similar.

(iii) It follows by the monotone method and the uniqueness of $U$ that $u \leq U \leq$ $\min \left\{1, \frac{1}{\alpha}\right\} z$. By the Lipschitz continuity of $g_{1}(S, R)$, there exists a constant $\bar{L}>\overline{0}$, such that $0 \leq g_{1}(z-u, z-\alpha u)-g_{1}(z-U, z-\alpha U) \leq L(U-u)$. Let $\hat{U}=U-u$. Then $\hat{U} \geq 0$ satisfies

$$
\begin{aligned}
& d \hat{U}_{x x}+\hat{U}\left[g_{1}(z-U, z-\alpha U)-u L\right] \leq 0, \quad 0<x<1, \\
& \hat{U}_{x}(0)=0, \quad \hat{U}_{x}(1)+\gamma \hat{U}(1)=0 .
\end{aligned}
$$

If $\hat{U} \not \equiv 0$, then the maximum principle leads to $\hat{U}>0$. Thus either $u<U$ or $u \equiv U$. The proof for $v$ is similar. 
Remark 2. It follows from Lemmas 1 and 2 that, for $\lambda_{i} \geq 1, i=1,2$, the only nonnegative solution of $(3)$ is $(0,0)$. In order to guarantee the existence of a positive solution of (3), we must assume that $\lambda_{i}<1$ for $i=1,2$.

Let $\hat{\lambda}_{i}$ be the principal eigenvalues and let $\hat{\phi}_{i}(x)>0, x \in[0,1], i=1,2$, be the corresponding eigenfunctions of the problem

$d \hat{\phi}_{1 x x}+\hat{\lambda}_{1} \hat{\phi}_{1} g_{1}(z-\beta V, z-V)=0, \quad 0<x<1, \quad \hat{\phi}_{1 x}(0)=0, \quad \hat{\phi}_{1 x}(1)+\gamma \hat{\phi}_{1}(1)=0$, $d \hat{\phi}_{2 x x}+\hat{\lambda}_{2} \hat{\phi}_{2} g_{2}(z-U, z-\alpha U)=0, \quad 0<x<1, \quad \hat{\phi}_{2 x}(0)=0, \quad \hat{\phi}_{2 x}(1)+\gamma \hat{\phi}_{2}(1)=0$.

Theorem 1. Suppose $\hat{\lambda}_{i}<1$ for $i=1,2$. Then there exists a positive steady-state solution $(u, v)$ of (3) satisfying $0<u(x)<U(x), 0<v(x)<V(x)$ for $x \in[0,1]$.

Proof. It is easy to check that $(U, V)$ is the sup-solution of (3). Let $(\underline{u}, \underline{v})=$ $\left(\delta \hat{\phi}_{1}, \delta \hat{\phi}_{2}\right)(\delta>0)$. Then for $\delta$ sufficiently small, we have

$$
\begin{aligned}
& d \underline{u}_{x x}+\underline{u} g_{1}(z-\underline{u}-\beta V, z-\alpha \underline{u}-V) \\
= & {\left[\underline{u} g_{1}(z-\underline{u}-\beta V, z-\alpha \underline{u}-V)-\hat{\lambda}_{1} \underline{u} g_{1}(z-\beta V, z-V)\right] } \\
= & \underline{u}\left[\left(1-\hat{\lambda}_{1}\right) g_{1}(z-\beta V, z-V)\right. \\
& \left.\quad+\left(g_{1}(z-\underline{u}-\beta V, z-\alpha \underline{u}-V)-g_{1}(z-\beta V, z-V)\right)\right]>0 .
\end{aligned}
$$

Hence there exists a solution $(u, v)$ of $(3)$ satisfying $\left(\delta \hat{\phi}_{1}, \delta \hat{\phi}_{2}\right) \leq(u, v) \leq(U, V)$ for small $\delta$. By Lemma 2 we obtain the strict inequalities in Theorem 1.

Now we consider the special case that $g_{1}=g_{2}=g$, and we find that there exist infinitely many positive solutions of (3).

Theorem 2. Suppose that $\lambda_{i}<1$ for $i=1,2$ and $g_{1}=g_{2}=g$. Then there exist infinitely many positive solutions $\left(u_{\rho}, v_{\rho}\right)(\rho>0)$ of (3) satisfying $0<v_{\rho} \leq$ $\min \left\{\frac{1}{\rho+\beta}, \frac{1}{\alpha \rho+1}\right\} z, u_{\rho}=\rho v_{\rho}$.

Proof. Set $\omega=\frac{u}{v}$. Then $\omega$ satisfies

$$
-d \omega_{x x}-\frac{2 d v_{x}}{v} \omega_{x}=0, \quad \omega_{x}(0)=\omega_{x}(1)=0 .
$$

By the maximum principle it follows that $\omega \equiv \rho$, a positive constant, i.e., $u=\rho v$. Thus $v$ satisfies

$$
d v_{x x}+v g(z-(\rho+\beta) v, z-(\alpha \rho+1) v)=0, \quad v_{x}(0)=0, \quad v_{x}(1)+\gamma v_{x}(1)=0 .
$$

For $\rho>0$ fixed, arguing as for the existence of $U$ or $V$, and noting that $\lambda_{2}<1$, it follows that there exists a unique positive solution of the above problem, say, $v_{\rho}$, satisfying $0<v_{\rho} \leq \min \left\{\frac{1}{\rho+\beta}, \frac{1}{\alpha \rho+1}\right\} z$. Thus $\left(u_{\rho}, v_{\rho}\right)(\rho>0)$, where $u_{\rho}=\rho v_{\rho}$, is the positive solution of (3). This completes the proof.

Remark 3. Suppose that $g_{1} \leq g_{2}, g_{1} \not \equiv g_{2}$ or $g_{1} \geq g_{2}, g_{1} \not \equiv g_{2}$. Then there exists no positive solution of (3). This conclusion is consistent with the analysis in [9] for the pure and simple competition model. In fact, suppose $u>0, v>0$ satisfy (3). We consider the first case, since the second case can be proved similarly. Denoting $\omega=\frac{u}{v}$, we have

$$
\begin{aligned}
& -d \omega_{x x}-\frac{2 d v_{x}}{v} \omega_{x}+\omega\left[g_{2}(z-u-\beta v, z-\alpha u-v)-g_{1}(z-u-\beta v, z-\alpha u-v)\right]=0, \\
& \omega_{x}(0)=\omega_{x}(1)=0 .
\end{aligned}
$$

Then $\omega=$ constant, and hence $\omega=0$, a contradiction. 
Theorem 3. Suppose $\lambda_{i}<1$ and $\hat{\lambda}_{i}>1$ for $i=1,2$. Then there exists a positive solution $(u, v)$ of $(3)$.

Proof. Let $C_{B}[0,1]=\left\{u(x) \in C[0,1]: u_{x}(0)=0, u_{x}(1)+\gamma u(1)=0\right\}$ be the Banach space, with the usual maximum norm, denoted by $\|\cdot\|, X=C_{B}[0,1] \times$ $C_{B}[0,1], \quad K=C_{B}^{+}[0,1] \times C_{B}^{+}[0,1]$, the positive cone of $X$. Let $N=(-d \Delta)^{-1}$, the inverse operator of $-d \Delta$ in $C_{B}[0,1]$. Then system (3) can be written as

$$
\begin{aligned}
& u-N\left(u g_{1}(z-u-\beta v, z-\alpha u-v)\right)=0, \\
& v-N\left(v g_{2}(z-u-\beta v, z-\alpha u-v)\right)=0 .
\end{aligned}
$$

Let $T(u, v)=\left(N\left(u g_{1}(z-u-\beta v, z-\alpha u-v)\right), N\left(v g_{2}(z-u-\beta v, z-\alpha u-v)\right)\right)$. Then the fixed points of $T$ in $K$ are the corresponding nonnegative solutions of (3). Define $D=\left\{(u, v) \in K:\|u\|+\|v\| \leq R_{0}\right\}$, where $R_{0}=2 \max \left\{1, \frac{1}{\alpha}, \frac{1}{\beta}\right\}\|z\|$, and let $\dot{D}$ denote the interior of $D$ in $K$. Since the proof is long, we divide it into three lemmas.

Lemma 3. For $\lambda \geq 1$, the equation $T(u, v)=\lambda(u, v)$ has no solution in $K$ satisfying $\|u\|+\|v\|=R_{0}$.

Proof. Suppose $(u, v) \in K$ satisfies $T(u, v)=\lambda(u, v)$. Then we have

$$
\begin{aligned}
& d u_{x x}+\lambda^{-1} u g_{1}(z-u-\beta v, z-\alpha u-v)=0, \\
& d v_{x x}+\lambda^{-1} v g_{2}(z-u-\beta v, z-\alpha u-v)=0,
\end{aligned}
$$

with the boundary conditions as above. As in the proof of Lemma 2, it follows that $u+\beta v<z, \alpha u+v<z$. Thus $u+v<\max \left\{1, \frac{1}{\alpha}, \frac{1}{\beta}\right\} z$. Hence there exists no fixed point of $T(u, v)=\lambda(u, v)$ in $K$ satisfying $\|u\|+\|v\|=R_{0}$.

Remark 4. It follows from Lemma 12.1 in [1] that $\operatorname{index}_{K}(T, \dot{D})=1$.

Let $P_{\sigma}(0,0)=\{(u, v) \in K:\|u\|+\|v\|<\sigma\}$ be the neighborhood of $(0,0)$ in $K$ with radius $\sigma$.

Lemma 4. For $\sigma>0$ small enough, index $\operatorname{de}_{K}\left(T, P_{\sigma}(0,0)\right)=0$.

Proof. Given $\epsilon_{0}>0$ sufficiently small, noting the definition of $U, V$, we can take $0<\sigma<\sigma_{0} \ll 1$ such that $\frac{\sigma}{\gamma}<\min \left\{U-\epsilon_{0}, V-\epsilon_{0}\right\}$. Denote $S_{\sigma}^{+}=\{(u, v) \in K$ : $\left.\|u\|+\|v\|=\frac{\sigma}{\gamma}\right\}$. Thus $\|u\| \leq \sigma z,\|v\| \leq \sigma z$ whenever $(u, v) \in S_{\sigma}^{+}$.

Let $\psi=(2+\gamma)-\gamma x^{2}$. Then $\psi>0$ on $[0,1]$ and satisfies

$$
\psi_{x x}<0, \quad 0<x<1, \quad \psi_{x}(0)=0, \quad \psi_{x}(1)+\gamma \psi(1)=0 .
$$

Take $p=(\psi, \psi)(\in K)$. We show next (by contradiction) that for $\lambda \geq 0,(u, v)-$ $T(u, v)=\lambda(\psi, \psi)$ has no solution on $S_{\sigma}^{+}$for small $\sigma$. Assume that this problem has a solution $(u, v)$ on $S_{\sigma}^{+}$. Then $(u, v)$ satisfies

$$
\begin{array}{ll}
d u_{x x}+u g_{1}(z-u-\beta v, z-\alpha u-v)=d \lambda \psi_{x x}, & 0<x<1 \\
d v_{x x}+v g_{2}(z-u-\beta v, z-\alpha u-v)=d \lambda \psi_{x x}, & 0<x<1 .
\end{array}
$$

Hence by the definition of $\psi$, we have

$$
\begin{array}{ll}
d u_{x x}+u g_{1}((1-\sigma \beta) z-u,(1-\sigma) z-\alpha u) \leq 0, & 0<x<1 \\
d v_{x x}+v g_{2}((1-\sigma) z-\beta v,(1-\sigma \alpha) z-v) \leq 0, & 0<x<1 .
\end{array}
$$

Since $\lambda_{i}<1$, we can take sufficiently small $\sigma$, say, $\sigma<\sigma_{1} \ll 1$, such that $\lambda_{1}\left(g_{1}((1-\right.$ $\sigma \beta) z,(1-\sigma) z))<1, \lambda_{2}\left(g_{2}((1-\sigma) z,(1-\sigma \alpha) z)\right)<1$, where $\lambda_{1}\left(g_{1}((1-\sigma \beta) z,(1-\right.$ $\sigma) z)), \lambda_{2}\left(g_{2}((1-\sigma) z,(1-\sigma \alpha) z)\right)$ are the principal eigenvalues of (4) with $g_{1}$ and $g_{2}$ 
replaced by $g_{1}((1-\sigma \beta) z,(1-\sigma) z)$ and $g_{2}((1-\sigma) z,(1-\sigma \alpha) z)$, respectively. As in the proof of Lemma 3.2 in [31] we can prove the existence and uniqueness of $U^{*}, V^{*}$ of the following problem:

$$
\begin{array}{ll}
d U_{x x}^{*}+U^{*} g_{1}\left((1-\sigma \beta) z-U^{*},(1-\sigma) z-\alpha U^{*}\right)=0, & 0<x<1, \\
d V_{x x}^{*}+V^{*} g_{2}\left((1-\sigma) z-\beta V^{*},(1-\sigma \alpha) z-V^{*}\right)=0, & 0<x<1,
\end{array}
$$

with the usual boundary conditions. By an $L^{p}$ estimate and the Sobolev embedding theorem (see [27]), we proceed as in the proof of Theorem 2.5 in [32] to obtain

$$
\lim _{\sigma \rightarrow 0} U^{*}=U, \lim _{\sigma \rightarrow 0} V^{*}=V .
$$

Thus there exists $\sigma_{2}>0$, such that for $\sigma<\sigma_{2}, U^{*}>U-\epsilon_{0}, V^{*}>V-\epsilon_{0}$. It follows from the monotone method and the uniqueness of $U^{*}, V^{*}$ that $u \geq U^{*}, v \geq V^{*}$. Now take $\sigma<\bar{\sigma}=\min \left\{\sigma_{0}, \sigma_{1}, \sigma_{2}\right\}$. Then for $\sigma<\bar{\sigma}$, we have $u>\frac{\sigma}{\gamma}, v>\frac{\sigma}{\gamma}$, which contradicts $(u, v) \in S_{\sigma}^{+}$. Lemma 12.1 of [1] can be applied to complete the proof of this lemma. lemma.

Let $O^{+}(U, 0)$ be a small neighborhood of $(U, 0)$ in $K$. Then we have the following

Lemma 5. Suppose that $T$ has no fixed point in $\dot{D}$. Then index $_{K}\left(T, O^{+}(U, 0)\right)=1$ if $\hat{\lambda}_{2}>1, \lambda_{1}<1$.

Proof. Define $T(\theta)(u, v)=\left(N\left(u g_{1}(z-u-\theta \beta v, z-\alpha u-\theta v)\right), N\left(v g_{2}(z-u-\right.\right.$ $\theta \beta v, z-\alpha u-\theta v)))$. It follows from $(u, v)=T(\theta)(u, v)$ that

$$
\begin{aligned}
& d u_{x x}+u g_{1}(z-u-\theta \beta v, z-\alpha u-\theta v)=0 \\
& d v_{x x}+v g_{2}(z-u-\theta \beta v, z-\alpha u-\theta v)=0 .
\end{aligned}
$$

If $(u, v)$ is a fixed point of $T(\theta)$ on $\partial O^{+}(U, 0)$, the boundary of $O^{+}(U, 0)$ in $K$, it is easy to see that $u>0, v \geq 0$. Furthermore, we have $v>0$; otherwise we have $(u, v)=(U, 0)$, contradicting $(u, v) \in \partial O^{+}(U, 0)$. We claim that for $\theta \in[0,1], T(\theta)$ has no fixed point on $\partial O^{+}(U, 0)$. Otherwise, for $\theta=0$, by noting $\hat{\lambda}_{2}>1$ and $\lambda_{1}<1$, we find $u=U, v=0$, a contradiction; for $\theta>0$, this implies that $(u, \theta v)>(0,0)$ is a fixed point of $T$ in $\dot{D}$, contradicting a hypothesis of this lemma. It follows from the homotopy invariance of topological degree that

$$
\operatorname{index}_{K}\left(T, O^{+}(U, 0)\right)=\operatorname{index}_{K}\left(T(1), O^{+}(U, 0)\right)=\operatorname{index}_{K}\left(T(0), O^{+}(U, 0)\right),
$$

where $T(0)(u, v)=\left(N\left(u g_{1}(z-u, z-\alpha u)\right), N\left(v g_{2}(z-u, z-\alpha u)\right)\right)$.

The fixed point $(u, v)$ of $T(0)$ in $O^{+}(U, 0)$ satisfies

$$
\begin{array}{ll}
d u_{x x}+u g_{1}(z-u, z-\alpha u)=0, & 0<x<1, \\
d v_{x x}+v g_{2}(z-u, z-\alpha u)=0, & 0<x<1,
\end{array}
$$

with the boundary conditions

$$
u_{x}(0)=0, \quad v_{x}(0)=0, \quad u_{x}(1)+\gamma u(1)=0, \quad v_{x}(1)+\gamma v(1)=0 .
$$

Since $\lambda_{1}<1$, we have $u=U$. Noting $\hat{\lambda}_{2}>1$, we determine that the principal eigenvalue $\lambda_{2}^{\prime}$ of the following problem is negative:

$$
d \phi_{x x}^{\prime}+\phi^{\prime} g_{2}(z-U, z-\alpha U)=\lambda_{2}^{\prime} \phi^{\prime}, \quad \phi_{x}^{\prime}(0)=0, \quad \phi_{x}^{\prime}(1)+\gamma \phi^{\prime}(1)=0 .
$$


Substituting $u=U$ into the second equation of (8), we have $v=0$. Hence $(U, 0)$ is the unique fixed point of $T(0)$ in $O^{+}(U, 0)$; thus

$$
\operatorname{index}_{K}\left(T(0), O^{+}(U, 0)\right)=\operatorname{index}_{K}(T(0),(U, 0)) .
$$

Let $I(\theta)(\theta \in[0,1])$ be defined by $I(\theta)(u, v)=\left(N\left(u g_{1}(z-u, z-\alpha u)\right), N\left(v g_{2}(z-\right.\right.$ $(\theta U+(1-\theta) u), z-\alpha(\theta U+(1-\theta) u))))$. Then $(u, v)=I(\theta)(u, v)$ satisfies

$$
\begin{array}{ll}
d u_{x x}+u g_{1}(z-u, z-\alpha u)=0, & 0<x<1, \\
d v_{x x}+v g_{2}(z-(\theta U+(1-\theta) u), z-\alpha(\theta U+(1-\theta) u))=0, & 0<x<1,
\end{array}
$$

with the usual boundary conditions. We claim that $I(\theta)$ has no fixed point on $\partial O^{+}(U, 0)$ in $K$. Otherwise, from the first equation of $(10)$, we have $u=U$, and substituting this into the second equation of (10), we find $v=0$, so the only fixed point of $I(\theta)$ on $\partial O^{+}(U, 0)$ is $(U, 0)$, a contradiction. By the definition of $I(\theta)$, we obtain

$$
T(0)=I(0), \quad I(1)=T_{1} \times T_{2},
$$

where $T_{1} u=N\left(u g_{1}(z-u, z-\alpha u)\right), T_{2} v=N\left(v g_{2}(z-U, z-\alpha U)\right),\left(T_{1} \times T_{2}\right)(u, v)=$ $\left(T_{1} u, T_{2} v\right) .(u, v)=I(1)(u, v)$ satisfies

$$
\begin{array}{ll}
d u_{x x}+u g_{1}(z-u, z-\alpha u)=0, & 0<x<1, \\
d v_{x x}+v g_{2}(z-U, z-\alpha U)=0, & 0<x<1 .
\end{array}
$$

It follows from (7)-(11) and the product theorem for fixed points (see [33]) that

$$
\begin{aligned}
\operatorname{index}_{K}(T(0),(U, 0)) & =\operatorname{index}_{K}(I(0),(U, 0))=\operatorname{index}_{K}(I(1),(U, 0)) \\
& =\operatorname{index}_{C_{B}}\left(T_{1}, U\right) \cdot \operatorname{index}_{C_{B}^{+}}\left(T_{2}, 0\right) .
\end{aligned}
$$

Since $T_{2}$ is a linear compact operator and $\hat{\lambda}_{2}>1$, then $T_{2}$ has no eigenvalue $>1$ with positive eigenfunction in $C_{B}^{+}$. It follows from Lemma 13.1 of [1] that index $C_{B}^{+}\left(T_{2}, 0\right)=$ 1.

We show next that index $_{C_{B}}\left(T_{1}, U\right)=1$. Let $\tau=2 \min \left\{1, \frac{1}{\alpha}\right\}\|z\|, P_{\tau}=\left\{u \in C_{B}^{+}\right.$: $\|u\| \leq \tau\}, \partial P_{\tau}=\left\{u \in C_{B}^{+}:\|u\|=\tau\right\}$. For $\lambda \geq 1$, if $T_{1} u=\lambda u, d \lambda u_{x x}+u g_{1}(z-$ $u, z-\alpha u)=0$. Arguing as in the proof of Lemma 1 , we have $u \leq \min \left\{1, \frac{1}{\alpha}\right\} z<\tau$. Hence for $\lambda \geq 1, T_{1} u=\lambda u$ has no solution on $\partial P_{\tau}$. It follows from Lemma 12.1 of [1] that index $C_{B}^{+}\left(T_{1}, P_{\tau}\right)=1$. Let $0<\tau_{0} \leq \frac{1}{2} \min _{[0,1]}\{U(x)\}$. Suppose that for $\lambda \geq 0, p=\psi(x)$, such that $u-T_{1} u=\lambda p$ has a solution $u$ on $\partial P_{\tau_{0}}$, where $\psi(x)$ is defined as in the proof of Lemma 4. Then, $d u_{x x}+u g_{1}(z-u, z-\alpha u)=d \lambda \psi_{x x} \leq 0$. Thus $u$ is a sup-solution of (5). From the monotone method and the uniqueness of $U$ it follows that $u \geq U$, a contradiction to $\|u\|=\tau_{0}$. Hence, index $C_{B}^{+}\left(T_{1}, P_{\tau_{0}}\right)=0$. Since $u=U$ is the unique fixed point of $T_{1}$ in $P_{\tau} \backslash \bar{P}_{\tau_{0}}$, we obtain index $_{C_{B}}\left(T_{1}, U\right)=$ index $_{C_{B}^{+}}\left(T_{1}, P_{\tau} \backslash \bar{P}_{\tau_{0}}\right)=$ index $_{C_{B}^{+}}\left(T_{1}, P_{\tau}\right)-$ index $_{C_{B}^{+}}\left(T_{1}, P_{\tau_{0}}\right)=1$.

Combining the above result with equations (7), (9), and (12), it follows that index $_{K}\left(T, O^{+}(U, 0)\right)=1$.

Remark 5. Suppose that $T$ has no fixed point in $\dot{D}$. We can proceed as above to obtain $\operatorname{index}_{K}\left(T, O^{+}(0, V)\right)=1$ if $\hat{\lambda}_{1}>1, \lambda_{2}<1$.

Now we turn to the proof of Theorem 3. Suppose that $T$ has no fixed point in $\dot{D}$. Then the following equation holds:

$\operatorname{index}_{K}(T, \dot{D})=\operatorname{index}_{K}\left(T, O^{+}(0,0)\right)+\operatorname{index}_{K}\left(T, O^{+}(U, 0)\right)+\operatorname{index}_{K}\left(T, O^{+}(0, V)\right)$, 
contradicting Lemmas 3-5. This completes the proof of Theorem 3.

Noting Lemma 1, and using the same process as in the proof of Theorem 3.6 in [15], we have the following theorem.

THEOREM 4. If $\lambda_{1}>1$ and $\lambda_{2}>1$, then the solution of system (1) satisfies

$$
\lim _{t \rightarrow \infty}(S, R, U, V)=(z, z, 0,0) .
$$

If $\lambda_{1}>1$ and $\lambda_{2}<1$, then the solution of system (1) satisfies

$$
\lim _{t \rightarrow \infty}(S, R, U, V)=(z-\beta V, z-V, 0, V) .
$$

If $\lambda_{1}<1$ and $\lambda_{2}>1$, then the solution of system (1) satisfies

$$
\lim _{t \rightarrow \infty}(S, R, U, V)=(z-U, z-\alpha U, U, 0) .
$$

TheOREM 5. Suppose $\hat{\lambda}_{i}<1, i=1,2$. Then the solution of system (1) is uniformly persistent ([10]).

Proof. It follows from system $\left(1^{\prime}\right)$ that $v(x, t) \leq V(x, t)$, where $V(x, t)$ is the solution of the problem

$$
\begin{aligned}
& V_{t}=d V_{x x}+V g_{2}\left(\varphi_{1}-\beta V, \varphi_{2}-V\right), \quad 0<x<1, \quad t>0, \\
& V_{x}(0, t)=0, \quad V_{x}(1, t)+\gamma V(1, t)=0, \\
& V(x, 0)=v_{0}(x) .
\end{aligned}
$$

Since $\hat{\lambda}_{2}<1$, then $\lambda_{2}<1$. We can proceed as in Theorem 2.5 in [32] to show that if $\lambda_{2}<1$, then $\lim _{t \rightarrow \infty} V(x, t)=V(x)$, where $V(x)<\min \left\{1, \frac{1}{\beta}\right\} z$ is the unique positive solution of

$$
\begin{aligned}
& d V_{x x}+V g_{2}(z-\beta V, z-V)=0, \quad 0<x<1, \\
& V_{x}(0)=0, \quad V_{x}(1)+\gamma V(1)=0 .
\end{aligned}
$$

${\underset{\tilde{\lambda}}{1}}_{1}<\hat{\lambda}_{1}<1$, we can take $0<\epsilon \ll 1$, such that for the following principal eigenvalue $\tilde{\lambda}_{1}<1$

$$
\begin{aligned}
& d \tilde{\phi}_{1 x x}+\tilde{\lambda}_{1} \tilde{\phi}_{1} g_{1}((1-\epsilon(1+\beta) / 2) z-\beta V(x),(1-\epsilon) z-V(x))=0, \quad 0<x<1, \\
& \tilde{\phi}_{1 x}(0)=0, \quad \tilde{\phi}_{1 x}(1)+\tilde{\phi}_{1}(1)=0 .
\end{aligned}
$$

There exists $\tau^{\prime}>0$ such that for $x \in[0,1], t \geq \tau^{\prime}$, the following inequalities hold:

$$
\varphi_{1} \geq z-(\epsilon / 2) z, \quad \varphi_{2} \geq z-(\epsilon / 2) z, \quad v(x, t) \leq V(x)+(\epsilon / 2) z .
$$

Using the comparison theorem, it follows that for $t \geq \tau^{\prime}, u(x, t) \geq \underline{u}(x, t)$, where $\underline{u}(x, t)$ is the solution of

$$
\begin{array}{lr}
\underline{u}_{t}=d \underline{u}_{x x}+\underline{u} g_{1}((1-(\epsilon(1+\beta) / 2)) z-\underline{u}-\beta V(x), & (1-\epsilon) z-\alpha \underline{u}-V(x)), \\
\underline{u}_{x}(0, t)=0, \quad \underline{u}_{x}(1, t)+\gamma \underline{u}(1, t)=0, & 0<x<1, t>\tau^{\prime}, \\
\underline{u}\left(x, \tau^{\prime}\right)=\min \{(1-\epsilon(1+\beta) / 2) z-\beta V(x), & \left.((1-\epsilon) z-V(x)) / \alpha, u\left(x, \tau^{\prime}\right)\right\} .
\end{array}
$$


Noting $\tilde{\lambda}_{1}<1$, we have $\lim _{t \rightarrow \infty} \underline{u}(x, t)=\underline{u}_{\epsilon}(x)$, where $\underline{u}_{\epsilon}(x)$ is the unique positive solution of

$$
d \underline{u}_{\epsilon x x}+\underline{u}_{\epsilon} g_{1}\left((1-\epsilon(1+\beta) / 2) z-\underline{u}_{\epsilon}-\beta V(x),(1-\epsilon) z-\alpha \underline{u}_{\epsilon}-V(x)\right)=0
$$

with the usual boundary conditions. It follows from an $L^{p}$ estimate and the embedding theorem (see [27]) that $\lim _{\epsilon \rightarrow 0} \underline{u}_{\epsilon}(x)=\hat{u}(x)$, where $\hat{u}(x)$ is the unique positive solution of the following problem on $[0,1]$ :

$$
d \hat{u}_{x x}+\hat{u} g_{1}(z-\hat{u}-\beta V(x), z-\alpha \hat{u}-V(x))=0
$$

with the usual boundary conditions. A similar result holds for $v$ if $\hat{\lambda}_{2}<1$. Hence, there exist constants $\eta_{1}>0, \tau_{1} \geq \tau^{\prime}$ such that $u(x, t) \geq \eta_{1}, v(x, t) \geq \eta_{1}$ for $x \in$ $[0,1], t \geq \tau_{1}$.

By the equation of $S$ in system (1) and the definition of $g_{i}, i=1,2$, we have

$$
\begin{aligned}
S_{t} & =d S_{x x}-g_{1}(S, R) u-\beta g_{2}(S, R) v \\
& \geq d S_{x x}-\max \left\{\frac{m_{s_{1}}}{K_{s_{1}}}, \frac{m_{s_{2}}}{K_{s_{2}}}\right\} S(u+\beta v) .
\end{aligned}
$$

Then there exists $\tau^{\prime \prime}>0$, and for $t \geq \tau^{\prime \prime}$, the following inequality holds:

$$
S_{t} \geq d S_{x x}-\max \left\{\frac{m_{s_{1}}}{K_{s_{1}}}, \frac{m_{s_{2}}}{K_{s_{2}}}\right\} S(z+\varepsilon-S) .
$$

A similar result holds for $R$. Thus we can proceed as in Lemma 3.8 in [15] to show that there exist $\eta_{2}>0, \tau_{2}>0$ such that $S(x, t) \geq \eta_{2}, R(x, t) \geq \eta_{2}$ for $x \in[0,1], t \geq \tau_{2}$. Denote $\tau=\max \left\{\tau_{1}, \tau_{2}\right\}, \eta=\max \left\{\eta_{1}, \eta_{2}\right\}$. Then we have $S \geq \eta, R \geq \eta, u \geq \eta, v \geq \eta$ for $x \in[0,1], t \geq \tau$. This completes the proof.

3. Numerical simulations. The goal of this section is to present the results of numerical simulations that complement the analytic results of the previous section. The simulations reported below represent a small fraction of those made. We wish to make a few general comments based on our observations. First, in most simulations performed, convergence to equilibrium was observed. Second, competitive exclusion, the elimination of one population by another, was observed. Finally, nonuniqueness of the positive equilibrium and bistability of the semitrivial equilibrium were observed. Our simulations are consistent with the analytic results of the previous sections. Furthermore, the simulations reveal that much more complicated dynamics are also possible in the region $D$ defined below. Our numerical simulations also seemed to indicate that coexistence is more likely in the case of competition for two limiting complementary resources in the unstirred chemostat, than in the case of competition for a single limiting resource in the unstirred chemostat (see [26]).

Define $A=\left\{\left(\hat{\lambda}_{1}, \hat{\lambda}_{2}\right): 0<\hat{\lambda}_{1}<1,0<\hat{\lambda}_{2}<1\right\}, B=\left\{\left(\hat{\lambda}_{1}, \hat{\lambda}_{2}\right): 0<\hat{\lambda}_{1}<1, \hat{\lambda}_{2}>\right.$ $1\}, C=\left\{\left(\hat{\lambda}_{1}, \hat{\lambda}_{2}\right): \hat{\lambda}_{1}>1,0<\hat{\lambda}_{2}<1\right\}$, and $D=\left\{\left(\hat{\lambda}_{1}, \hat{\lambda}_{2}\right): \hat{\lambda}_{1}>1, \hat{\lambda}_{2}>1\right\}$.

Our numerical simulations seem to indicate the following:

(1) Coexistence in the form of a positive equilibrium can be observed when $\left(\hat{\lambda}_{1}, \hat{\lambda}_{2}\right) \in A \cup B \cup C$ (see Figures 1-2 and Tables 1-2), and apparently a globally stable positive equilibrium can always be observed when $\left(\hat{\lambda}_{1}, \hat{\lambda}_{2}\right) \in A$;

(2) Competitive exclusion in the form of an apparently globally stable semitrivial positive equilibrium can occur when $\left(\hat{\lambda}_{1}, \hat{\lambda}_{2}\right) \in B \cup C$ (see Figures 1-2 and Tables 1-2); 
(3) Both stable and unstable positive equilibria can exist, and there can be bistability with two stable semitrivial equilibria and an unstable positive equilibrium, resulting in initial condition dependent outcomes when $\left(\hat{\lambda}_{1}, \hat{\lambda}_{2}\right) \in D$ (see Figures 3-4);

(4) Existence of multiple stable and/or unstable positive equilibria can be observed when $\left(\hat{\lambda}_{1}, \hat{\lambda}_{2}\right) \in D$ (see Figures $\left.4-5\right)$;

(5) The parameters have an apparent effect on the density of both organisms, i.e., the density $u$ can be nondecreasing and the density $v$ can be nonincreasing as $\alpha$ increases (see Figures 6(a)-6(c)). Similar results for $v$ and $u$ hold as $\beta$ increases. But the density of both organisms can decrease as $\gamma$ increases (see Figures 6(b) and 6(d)).

Now we describe an indirect method used for determining either $\hat{\lambda}_{i}>1$ or $\hat{\lambda}_{i}<1$ from numerical simulations. The method will be described for determining the sign of $\hat{\lambda}_{1}-1$ only, since the other case is similar. Consider the following system:

$$
\begin{array}{ll}
u_{t}=d u_{x x}+u g_{1}(z-u-\beta v, z-\alpha u-v), & 0<x<1, t>0, \\
v_{t}=d v_{x x}+v g_{2}(z-\beta v, z-v), & 0<x<1, t>0, \\
u_{x}(0, t)=0, \quad u_{x}(1, t)+\gamma u(1, t)=0, & \\
v_{x}(0, t)=0, \quad v_{x}(1, t)+\gamma v(1, t)=0, & \\
u(x, 0)=u_{0}(x) \geq 0, \quad v(x, 0)=v_{0}(x) \geq 0, \not \equiv 0, &
\end{array}
$$

where $u_{0}+\beta v_{0} \leq z, \alpha u_{0}+v_{0} \leq z$. Taking initial conditions characterized by a very small density of $u_{0}$, we can prove and observe numerically that $v$ rapidly approaches the equilibrium $V(x)$. Hence for large times, $t$, we take $v(x, t)$ as $V(x)$ in the first equation of (14). Then we have

$$
u_{t}=d u_{x x}+u g_{1}(z-u-\beta V, z-\alpha u-V), \quad 0<x<1, t>0
$$

with the usual boundary and initial conditions. We can use the comparison theorem and the Liapunov function method to prove that the solution $u(x, t)$ of $(15)$ satisfies $\lim _{t \rightarrow \infty} u(x, t)=0$ if $\hat{\lambda}_{1} \geq 1$ and $\lim _{t \rightarrow \infty} u(x, t)=\hat{u}$ if $\hat{\lambda}_{1}<1$, where $\hat{u}$ is the unique positive solution of (13). Therefore, what happens to $u$ depends essentially on the sign of $\hat{\lambda}_{1}-1$. If $\hat{\lambda}_{1} \geq 1$, we observed the decay of the solution $u$ of (15) to very small values; if $\hat{\lambda}_{1}<1$, we observed the growth of the solution $u$ of (15) to the value of the solution of (13). Therefore, we can determine the sign of $\hat{\lambda}_{1}-1$ numerically by observing whether there is decay to very small values or growth to the value of the solution of (13).

We next simulate the corresponding time-dependent system of (3), which determines the limiting system of (1):

$$
\begin{array}{ll}
u_{t}=d u_{x x}+u g_{1}(z-u-\beta v, z-\alpha u-v), & 0<x<1, t>0, \\
v_{t}=d v_{x x}+v g_{2}(z-u-\beta v, z-\alpha u-v), & 0<x<1, t>0, \\
u_{x}(0, t)=0, \quad u_{x}(1, t)+\gamma u(1, t)=0, & \\
v_{x}(0, t)=0, v_{x}(1, t)+\gamma v(1, t)=0, & \\
u(x, 0)=u_{0}(x) \geq 0, v(x, 0)=v_{0}(x) \geq 0 . &
\end{array}
$$

We have chosen to discretize the spatial variables in the above system using a second-order finite-difference scheme. The derivative terms in the boundary conditions are approximated using second-order centered differencing. The temporal variable is approximated using the Crank-Nicholson method. In all of the simulations the domain is divided uniformly into 40 cells. 
TABLE 1 and 2 .

The equilibria corresponding to the parameters given in Tables 1 and 2 are shown in Figures 1

\begin{tabular}{|c|c|c|c|c|}
\hline$m_{s}$ & $\hat{\lambda}_{1}$ & $\hat{\lambda}_{2}$ & Area & Equilibrium \\
\hline$(2.06,2.04)$ & $<1$ & $>1$ & $\mathrm{~B}$ & $(U, 0)$ \\
\hline$(2.045,2.055)$ & $<1$ & $>1$ & $\mathrm{~B}$ & Coexistence \\
\hline$(2.04,2.06)$ & $<1$ & $<1$ & $\mathrm{~A}$ & Coexistence \\
\hline$(1.8,2.3)$ & $<1$ & $<1$ & $\mathrm{~A}$ & Coexistence \\
\hline$(1.6,2.5)$ & $<1$ & $<1$ & $\mathrm{~A}$ & Coexistence \\
\hline$(1.55,2.55)$ & $>1$ & $<1$ & $\mathrm{C}$ & $(0, V)$ \\
\hline
\end{tabular}

TABLE 2

\begin{tabular}{|c|c|c|c|c|}
\hline$m_{s}$ & $\hat{\lambda}_{1}$ & $\hat{\lambda}_{2}$ & Area & Equilibrium \\
\hline$(4.55,4.45)$ & $<1$ & $>1$ & $\mathrm{~B}$ & $(U, 0)$ \\
\hline$(4.48,4.52)$ & $<1$ & $>1$ & $\mathrm{~B}$ & Coexistence \\
\hline$(4.45,4.55)$ & $<1$ & $<1$ & $\mathrm{~A}$ & Coexistence \\
\hline$(2.2,6.8)$ & $<1$ & $<1$ & $\mathrm{~A}$ & Coexistence \\
\hline$(2.1,6.9)$ & $>1$ & $<1$ & $\mathrm{C}$ & Coexistence \\
\hline$(1.95,7.05)$ & $>1$ & $<1$ & $\mathrm{C}$ & $(0, V)$ \\
\hline
\end{tabular}

In the following, we denote $m_{s}=\left(m_{s_{1}}, m_{s_{2}}\right), m_{r}=\left(m_{r_{1}}, m_{r_{2}}\right), K_{s}=\left(K_{s_{1}}, K_{s_{2}}\right)$, $K_{r}=\left(K_{r_{1}}, K_{r_{2}}\right)$.

Coexistence and competitive exclusion. In Figures 1 and 2 a sequence of simulations is reported where different growth rates were used, but all of the other parameter values remain fixed. The parameter values used were $\alpha=\beta=0.5, \gamma=1, K_{s}=$ $(1,1), K_{r}=(1,1.2)$. In Figure 1 and Table $1, m_{r}=(3,3)$, and in Figure 2 and Table 2, $m_{r}=(6,6)$. In Figure 1, $m_{s}$ took the values indicated in Table 1, and in Figure 2, $m_{s}$ took the values indicated in Table 2. In each simulation in Figures 1 and 2 , the densities were plotted at the final time, $t=1000$. This appeared to be long enough to allow the solutions to be very close to steady state. A similar procedure was used in the other figures. We observed from Figures 1 and 2, as well as from many other simulations, that at the highest growth rate of $u$ and the lowest growth rate of $v, u$ is dominant with $v$ barely present for any initial conditions. In this case, we checked that $\left(\hat{\lambda}_{1}, \hat{\lambda}_{2}\right) \in B$. As the growth rate of $u$ is decreased or the growth rate of $v$ is increased, the amount of $v$ increases at the expense of the amount of $u$. Both organisms coexist at a positive equilibrium. In this case, we also checked that $\left(\hat{\lambda}_{1}, \hat{\lambda}_{2}\right) \in A$ or $B$ or $C$. All the simulations show that the coexistence is unique and an apparently globally stable positive equilibrium exists when $\left(\hat{\lambda}_{1}, \hat{\lambda}_{2}\right) \in A$. As the growth rate of $u$ is further decreased or the growth rate of $v$ is further increased, $v$ is dominant with $u$ barely present for any initial condition. In addition, we checked $\left(\hat{\lambda}_{1}, \hat{\lambda}_{2}\right) \in C$ in this case. Coexistence in the form of a positive equilibrium can occur when $\left(\hat{\lambda}_{1}, \hat{\lambda}_{2}\right) \in A$ or $B$ or $C$. The nonexistence of a positive equilibrium can also occur when $\left(\hat{\lambda}_{1}, \hat{\lambda}_{2}\right) \in B$ or $C$.

Bistability and the existence of positive equilibria. (i) In Figure 3, we provide numerical evidence of bistability; i.e., each of the two semitrivial equilibria is stable to invasion by its rival and attracts solutions corresponding to nearby initial data. As well, an unstable positive equilibrium is observed. We took $m_{s}=(3,2), m_{r}=$ $(2.4,3.6)$, and the other parameter values as in Figure 1. In this case we checked that $\left(\hat{\lambda}_{1}, \hat{\lambda}_{2}\right) \in D$. The simulations in Figures $3(\mathrm{a})$ and $3(\mathrm{~b})$ show a plot of the $L_{1}$ norms of $u$ and of $v$ versus time t. In Figure 3(a) the initial conditions used were $u_{0}=0.5$ and 


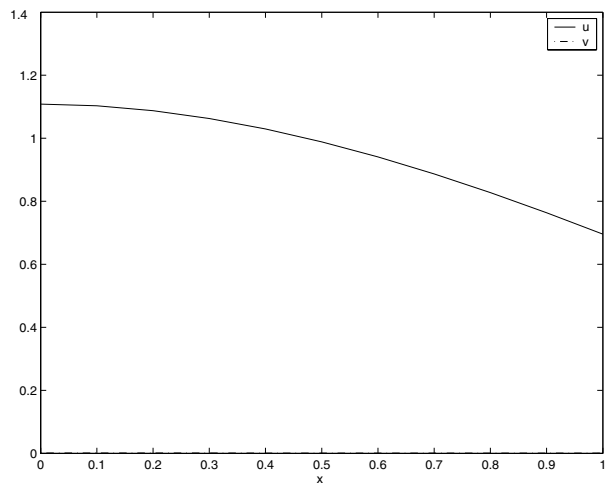

(a)

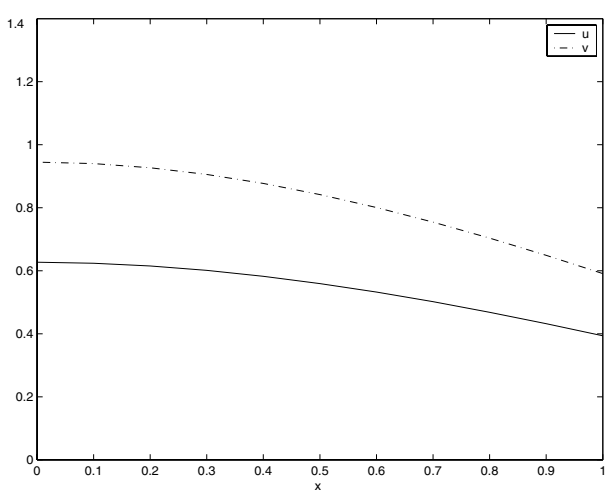

(c)

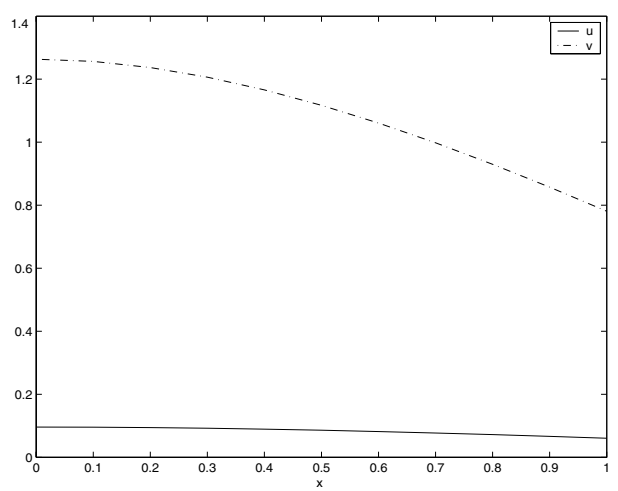

(e)

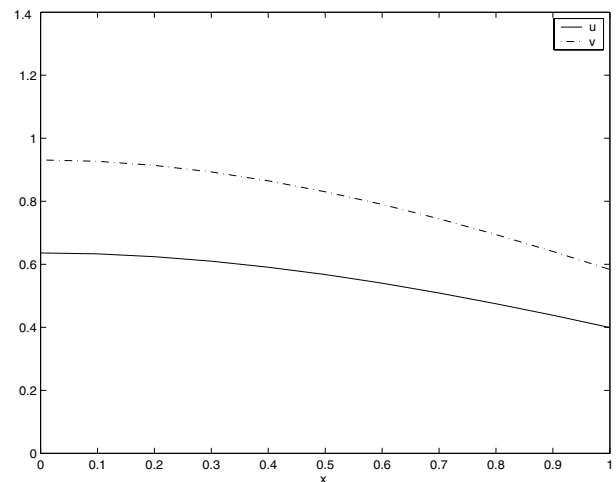

(b)

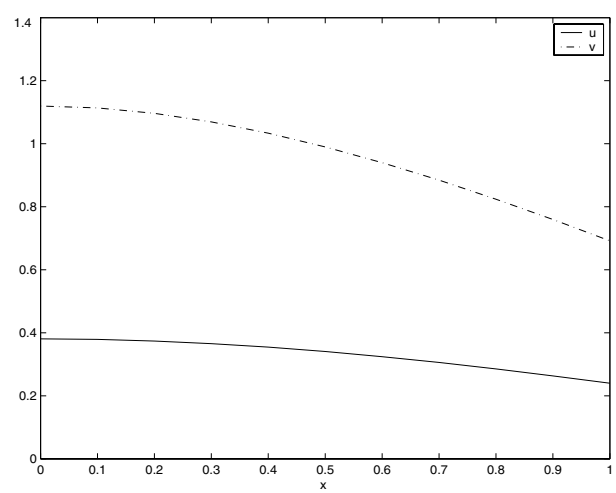

(d)

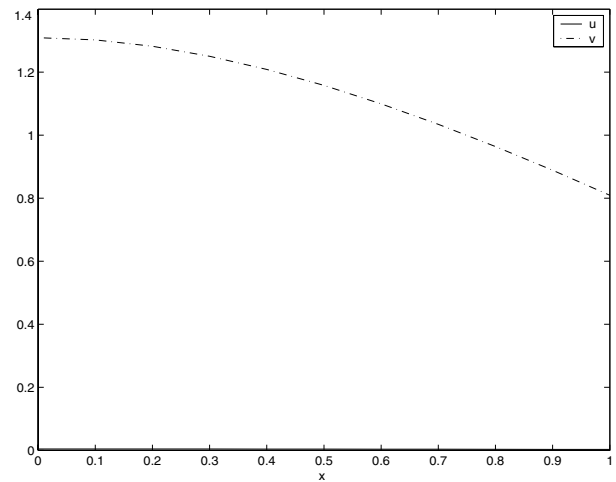

(f)

FIG. 1. Equilibria for $m_{r}=(3,3)$ and the different values of $m_{s}$ from Table 1 (in the order given in that table). The other parameters used are $K_{s}=(1,1), K_{r}=(1,1.2), \alpha=\beta=0.5$, and $\gamma=1$. 


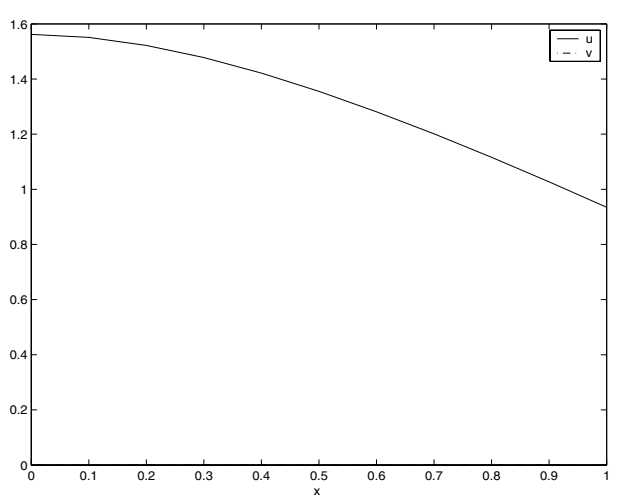

(a)

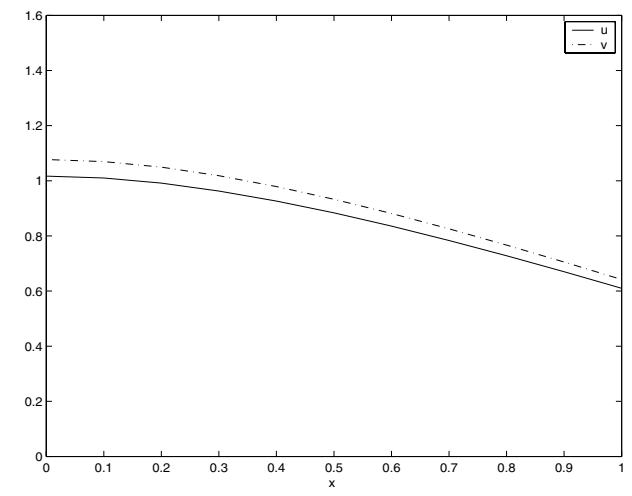

(c)

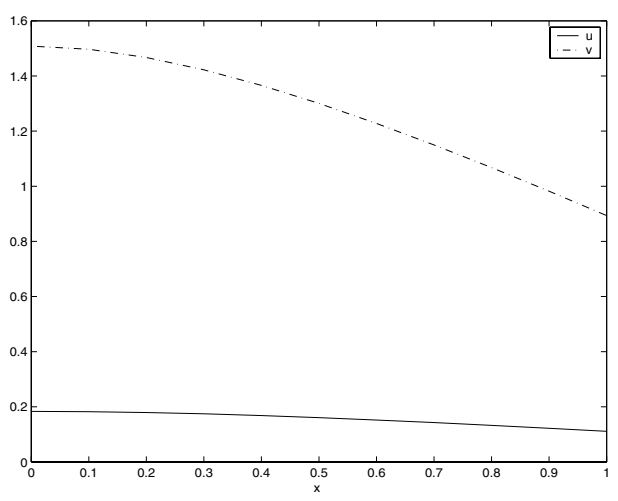

(e)

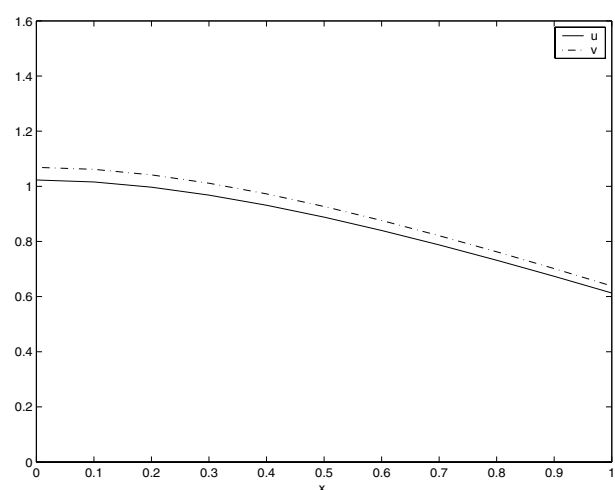

(b)

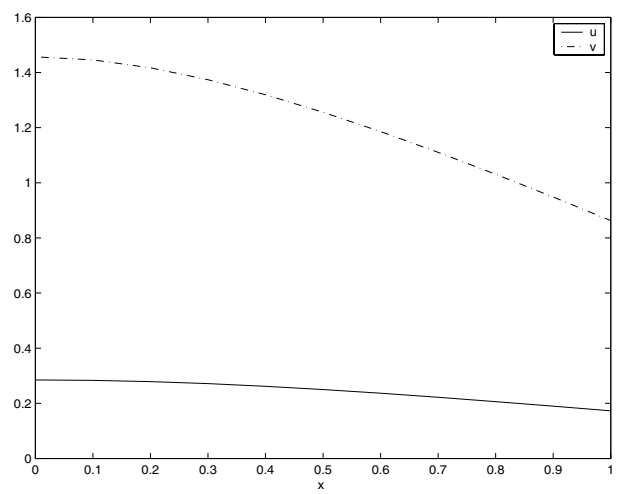

(d)

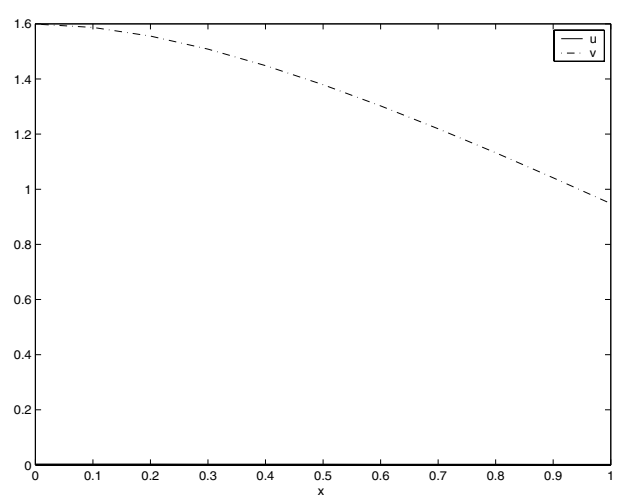

(f)

FIG. 2. Equilibria for $m_{r}=(6,6)$ and the different values of $m_{s}$ from Table 2 (in the order given in that table). All the other parameters are the same as those in Figure 1. 


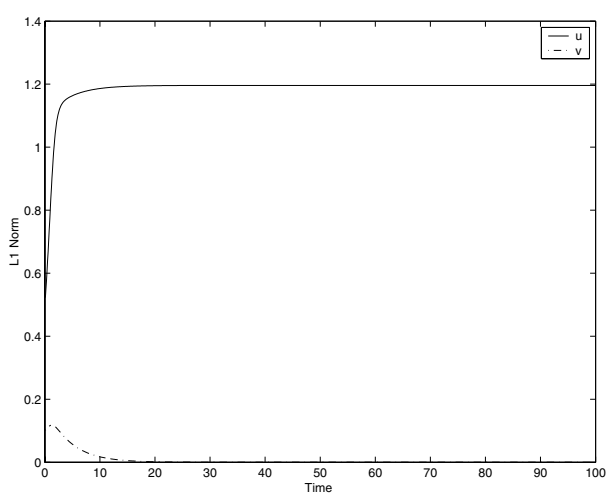

(a)

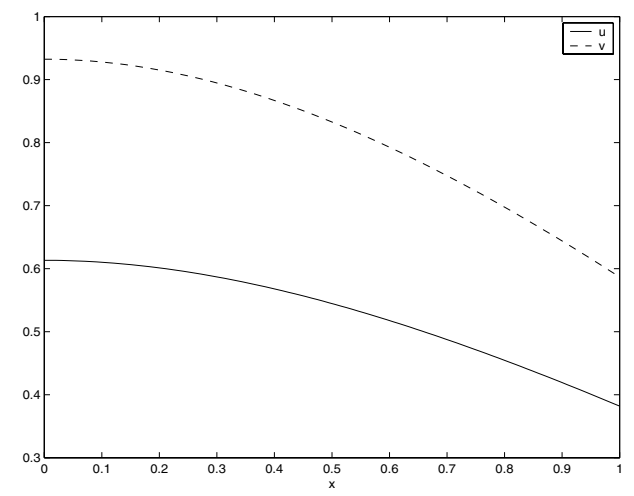

(c)

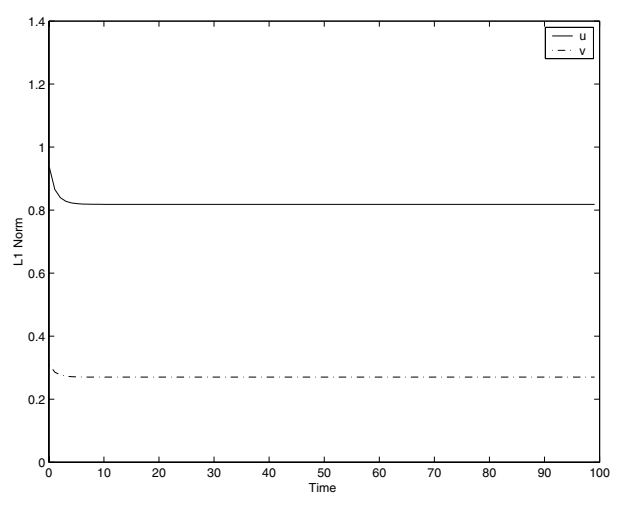

(e)

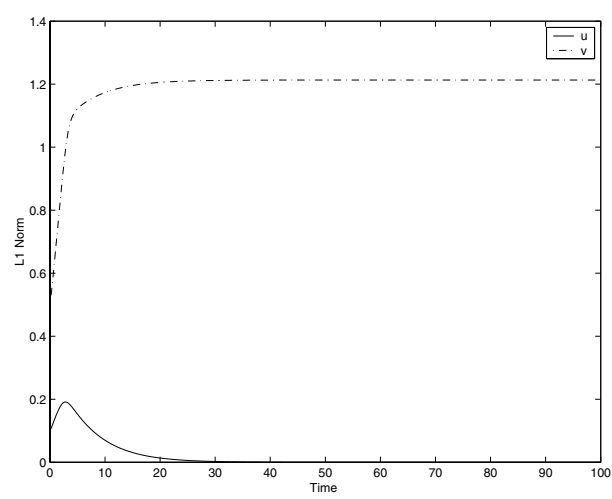

(b)

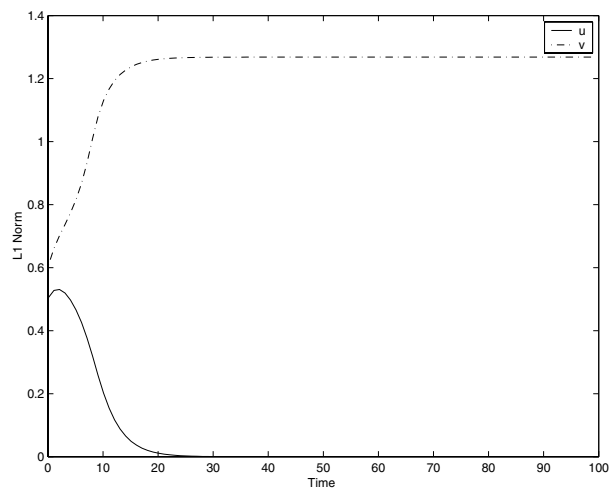

(d)

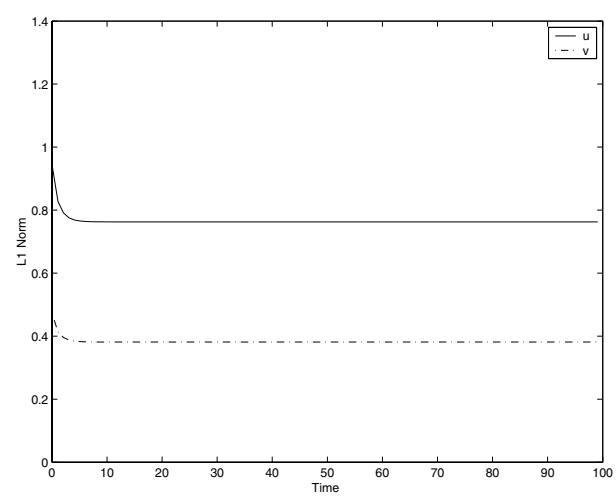

(f)

FIG. 3. Convergence to equilibria. All parameters except $m_{s}$ and $m_{r}$ are the same as those in Figure 1. In (a)-(c) $m_{s}=(3,2)$ and $m_{r}=(2.4,3.6)$. In (a)-(b) the $L_{1}$ norms of $u$ and of $v$ versus time are shown for two semitrivial equilibria. In (c) a plot of the positive equilibrium for each $x \in[0,1]$ is shown. In (d)-(f) $m_{s}=(2,2)$ and $m_{r}=(2,4)$. The $L_{1}$ norms of $u$ and of $v$ versus time are shown for several different equilibria. 


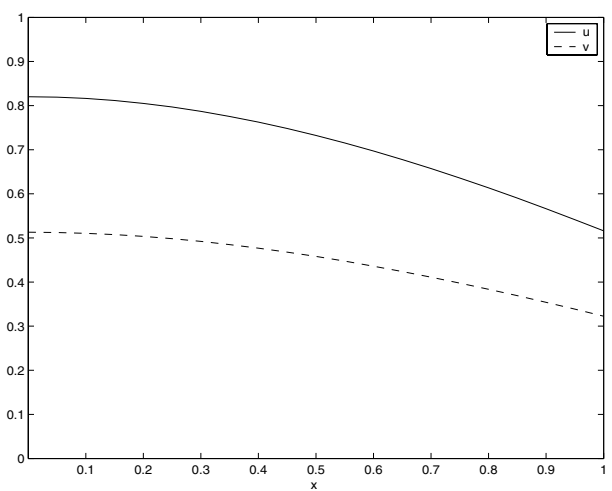

(a)

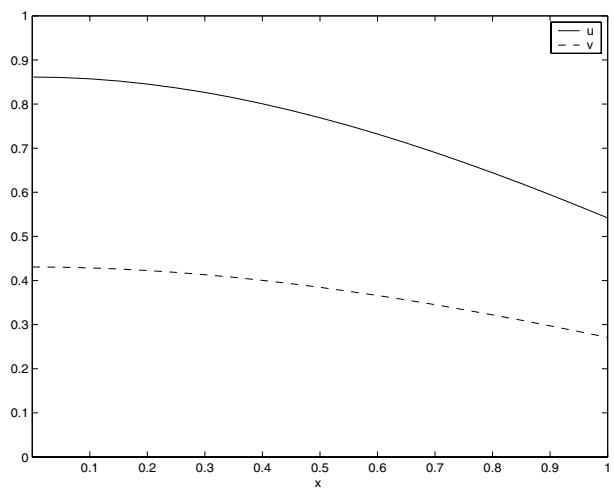

(c)

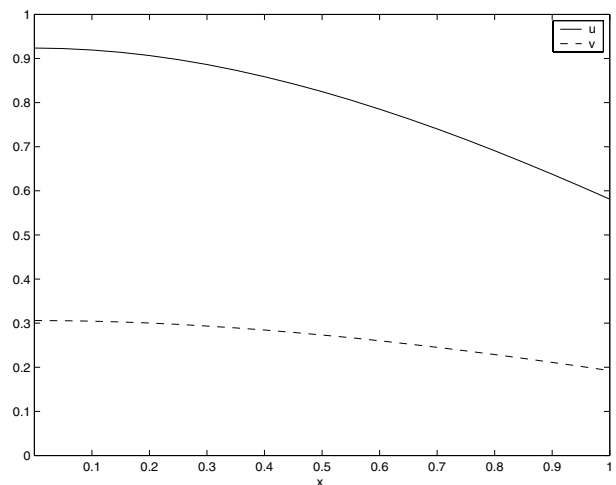

(b)

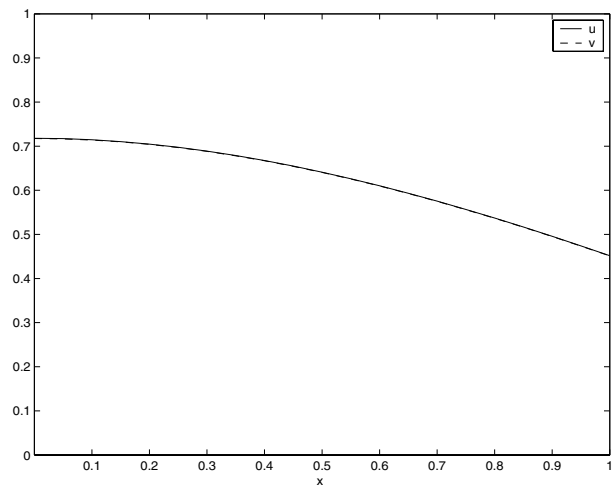

(d)

FIG. 4. Several positive equilibria. All parameters are the same as those in Figure 1, except $m_{s}=(2,2)$ and $m_{r}=(2,4)$. Note that $u$ and $v$ are indistinguishable in $(\mathrm{d})$.

$v_{0}=0.1$. In Figure $3(\mathrm{~b})$ the initial conditions used were $u_{0}=0.1$ and $v_{0}=0.5$. The positive equilibrium is plotted in Figure 3(c). After many simulations, in this case we believe that this is the only positive equilibrium and that it is unstable.

(ii) In Figures 3(d)-(f), we took $m_{s}=(2,2)$ and $m_{r}=(2,4)$. All other parameters are the same as in Figure 1. Both semitrivial equilibria are stable. Only one of them is shown (see Figure 3(d)). As well, nonuniqueness and stability of more than one positive equilibrium are observed. In this case, we checked that $\left(\hat{\lambda}_{1}, \hat{\lambda}_{2}\right) \in D$.

Existence of multiple positive equilibria. Based on extensive simulations, we believe that much more complicated dynamical behavior can occur when $\left(\hat{\lambda}_{1}, \hat{\lambda}_{2}\right) \in D$.

(i) In Figure 4 we took the same parameters as in Figures 3(d)-(f) and used continuation (numerical analysis) to find the equilibria. Simulations (not shown) seem to indicate that there are at least four positive equilibria in this case, and strongly suggest that one of them is unstable (see Figure 4(d), where $u$ and $v$ are indistinguishable), and that the other three are stable (see Figures $4(\mathrm{a})-(\mathrm{c})$ ). Note that the equilibria depicted in Figures 4(b)-(c) correspond to those in Figures 3(e)-(f), respectively.

(ii) In Figures 5(a)-(c) we took $m_{s}=(1.5,1.8)$ and $m_{r}=(1.75,1.42)$. In Figures $5(\mathrm{~d})-(\mathrm{f})$ we took $m_{s}=(2.1,2.75)$ and $m_{r}=(2.8,2.13)$. The other parameters 


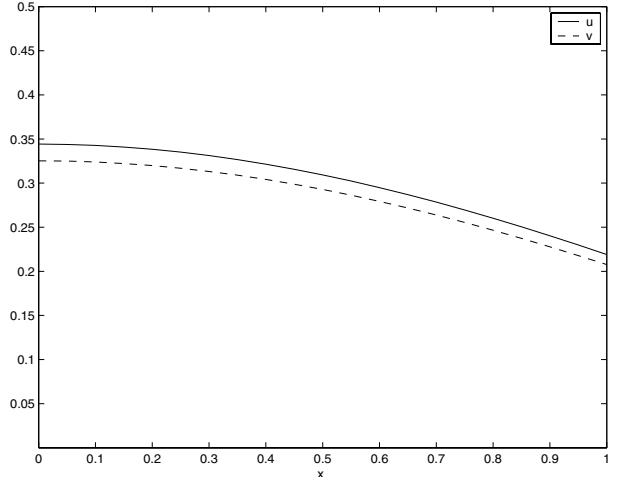

(a)

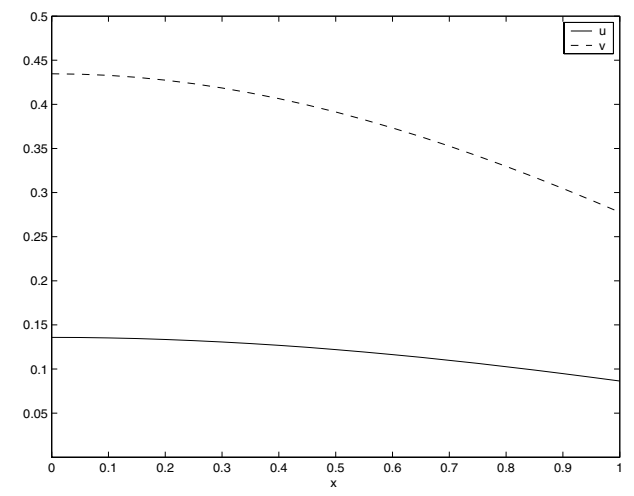

(c)

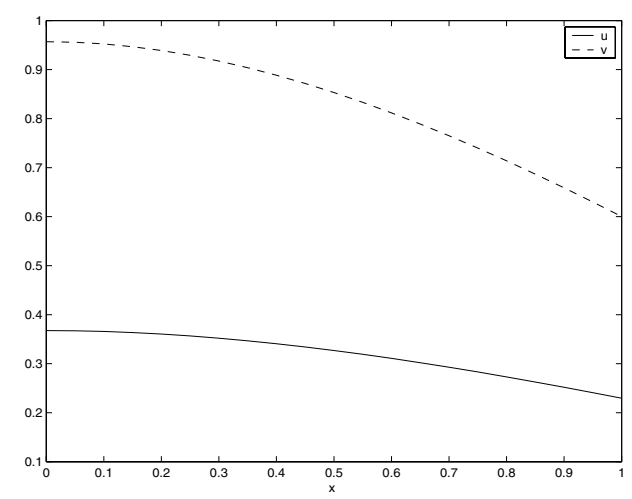

(e)

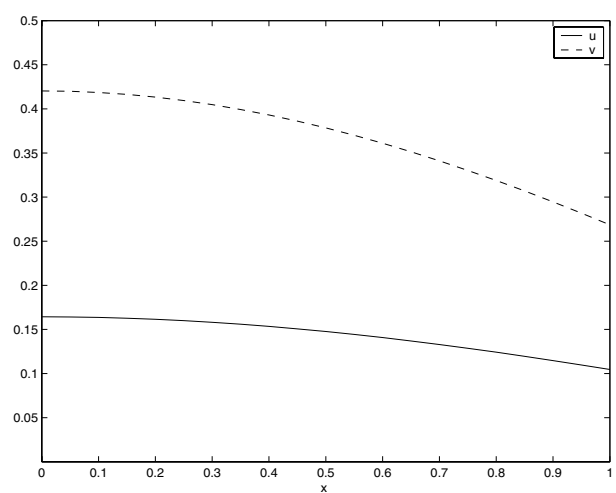

(b)

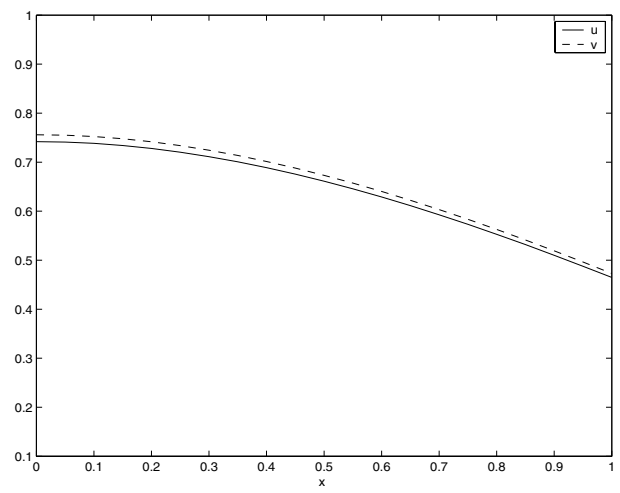

(d)

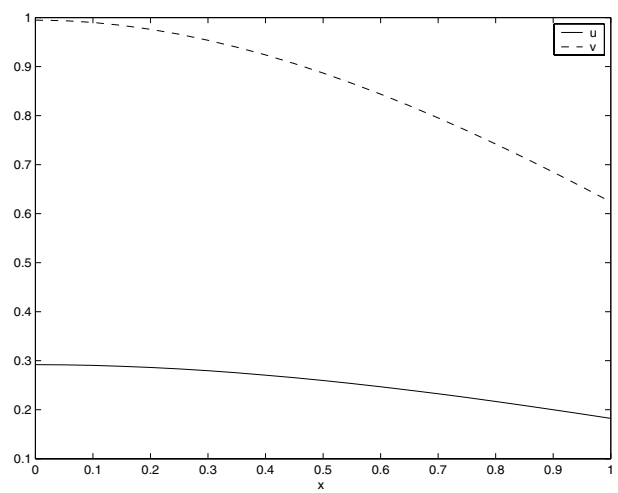

(f)

FIG. 5. Positive equilibria. All parameters are the same as those in Figure 1, except that in (a) - (c) $m_{s}=(1.5,1.8)$ and $m_{r}=(1.75,1.42)$, and in (d) $-(\mathrm{f}) m_{s}=(2.1,2.75)$ and $m_{r}=(2.8,2.13)$. 


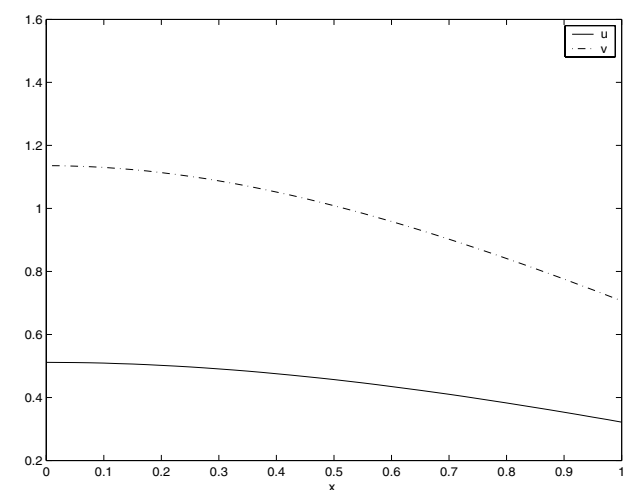

(a)

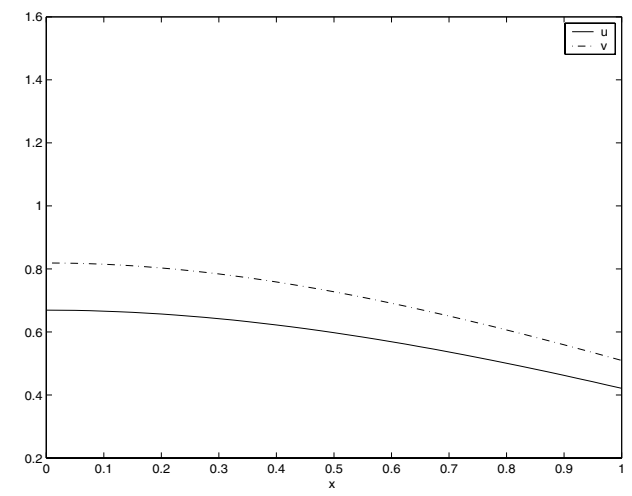

(c)

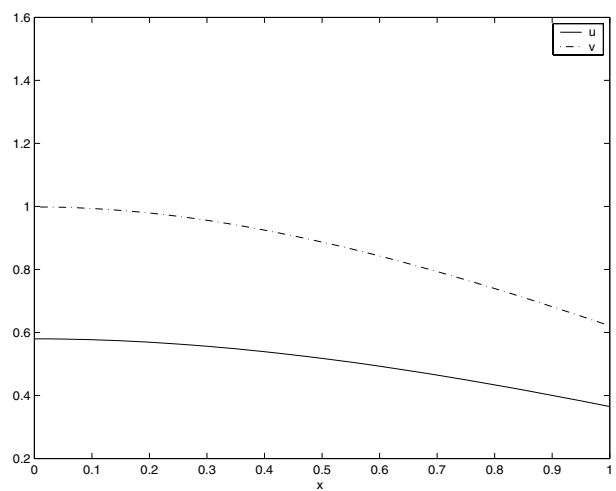

(b)

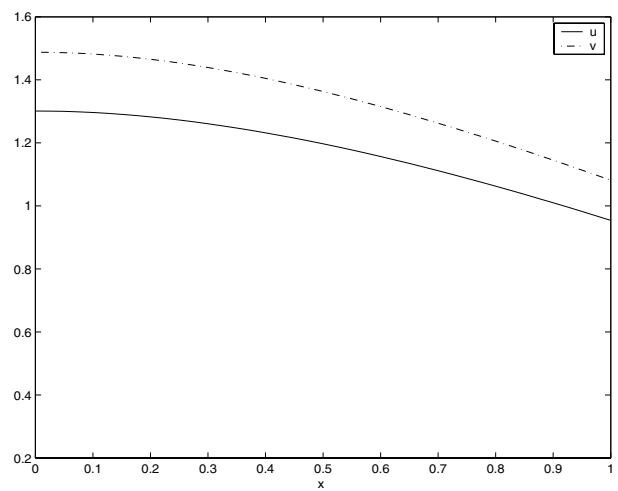

(d)

FIG. 6. Parameters $\alpha$ and $\gamma$ have an effect on the densities of the equilibria. In (a)-(d) $m_{s}=(2,2.1), m_{r}=(3,3), K_{s}=(1,1), K_{r}=(1,1.2)$, and $\beta=0.5$. In (a)-(c) $\gamma=1$, and in (d) $\gamma=0.6$. In (a) $\alpha=0.3$, in (b) $\alpha=0.5$, in (c) $\alpha=0.7$, and in (d) $\alpha=0.5$.

were taken as in Figure 1. In both cases, we checked that $\left(\hat{\lambda}_{1}, \hat{\lambda}_{2}\right) \in D$. For each case, we used numerical analysis to find the three positive equilibria depicted in Figure 5. Subsequent simulations strongly indicated that all these positive equilibria are unstable.

Effects of the parameters. In Figure 6 a sequence of simulations shows that the parameters $\alpha, \beta, \gamma$ have an apparent effect on the density of both populations. Parameter values taken are $m_{s}=(2,2.1), m_{r}=(3,3), K_{s}=(1,1), K_{r}=(1,1.2)$. Values for $\alpha, \beta$, and $\gamma$ are given in the caption of Figure 6. The initial data are $u_{0}=1$ and $v_{0}=1$. We observed that the density of $u$ can be nondecreasing and the density of $v$ can be nonincreasing as $\alpha$ increases (see Figures 6(a)-6(c)). A similar result holds for $v$ and $u$ as $\beta$ increases. We also observed that the density of both $u$ and $v$ can decrease as $\gamma$ increases (see Figures 6(b) and 6(d)).

Acknowledgment. The authors would like to express their sincere thanks to the anonymous referees of this paper for their careful reading and valuable suggestions leading to an improvement of the paper. 


\section{REFERENCES}

[1] H. Amann, Fixed point equations and nonlinear eigenvalue problems in ordered Banach spaces, SIAM Rev., 18 (1976), pp. 620-709.

[2] M. M. Ballyk And G. S. K. Wolkowicz, An examination of the thresholds of enrichment: A resource-based growth model, J. Math. Biol., 33 (1995), pp. 435-457.

[3] M. M. Ballyk and G. S. K. Wolkowicz, Exploitative competition in the chemostat for two perfectly substitutable resources, Math. Biosci., 118 (1993), pp. 127-180.

[4] B. C. Baltzis And A. G. Fredrickson, Limitation of growth rate by two complementary nutrients: Some elementary and neglected considerations, Biotechnol. Bioeng., 31 (1988), pp. $75-86$.

[5] J. V. BaXley and H. B. Thompson, Nonlinear boundary value problems and competition in the chemostat, Nonlinear Anal., 22 (1994), pp. 1329-1344.

[6] G. J. Butler And G. S. K. Wolkowicz, A mathematical model of the chemostat with a general class of functions describing nutrient uptake, SIAM J. Appl. Math., 45 (1985), pp. $138-151$.

[7] G. J. Butler and G. S. K. Wolkowicz, Exploitative competition in a chemostat for two complementary, and possibly inhibitory, resources, Math. Biosci., 83 (1987), pp. 1-48.

[8] L. Dung And H. L. Smith, A parabolic system modelling microbial competition in an unmixed bio-reactor, J. Differential Equations, 130 (1996), pp. 59-91.

[9] A. G. Fredrickson and G. Stephanopoulos, Microbial competition, Science, 213 (1981), pp. 972-979.

[10] J. Hale And P. Waltman, Persistence in infinite-dimensional systems, SIAM J. Math. Anal., 20 (1989), pp. 388-395.

[11] S. R. Hansen ANd S. P. Hubbell, Single nutrient microbial competition: Agreement between experimental and theoretical forecast outcomes, Science, 207 (1980), pp. 1491-1493.

[12] W. Harder And L. Dijkhuizen, Strategies of mixed substrate utilization in microorganisms, Philos. Trans. R. Soc. London B., 297 (1982), pp. 459-480.

[13] S.-B. Hsu, K.-S. Cheng, AND S. P. Hubbell, Exploitative competition of microorganisms for two complementary nutrients in continuous cultures, SIAM J. Appl. Math., 41 (1981), pp. $422-444$.

[14] S. B. Hsu, S. Hubbell, and P. Waltman, A mathematical theory for single-nutrient competition in continuous cultures of micro-organisms, SIAM J. Appl. Math., 32 (1977), pp. 366383.

[15] S.-B. Hsu AND P. Waltman, On a system of reaction-diffusion equations arising from competition in an unstirred chemostat, SIAM J. Appl. Math., 53 (1993), pp. 1026-1044.

[16] S. B. Hsu, H. L. Smith, And P. Waltman, Dynamics of competition in the unstirred chemostat, Canad. Appl. Math. Quart., 2 (1994), pp. 461-483.

[17] J. A. Leon And D. B. Tumpson, Competition between two species for two complementary or substitutable resources, J. Theoret. Biol., 50 (1975), pp. 185-201.

[18] B. Li And H. Smith, How many species can two essential resources support?, SIAM J. Appl. Math., 62 (2001), pp. 336-366.

[19] B. Li, G. S. K. Wolkowicz, AND Y. KUANG, Global asymptotic behavior of a chemostat model with two perfectly complementary resources and distributed delay, SIAM J. Appl. Math., 60 (2000), pp. 2058-2086.

[20] S. S. Pilyugin and P. Waltman, Competition in the unstirred chemostat with periodic input and washout, SIAM J. Appl. Math., 59 (1999), pp. 1157-1177.

[21] M. H. Protter and H. F. Weinberger, Maximum Principle in Differential Equations, Springer-Verlag, New York, 1984.

[22] D. J. RAPPorT, An optimization model of food selection, Am. Nat., 105 (1971), pp. 575-587.

[23] H. L. Smith and P. Waltman, Competition in an unstirred multi-dimensional chemostat, in Differential Equations and Applications to Biology and to Industry (Claremont, CA, 1994), M. Martelli et al., eds., World Scientific, River Edge, NJ, 1996, pp. 475-486.

[24] H. L. Sмiтh, An application of monotone systems theory to a model of microbial competition, in Differential Equations and Control Theory (Wuhan, 1994), Lecture Notes in Pure and Appl. Math. 176, Z. Deng et al., eds., Marcel Dekker, New York, pp. 293-307.

[25] H. L. Smith and P. Waltman, The Theory of the Chemostat, Cambridge University Press, Cambridge, UK, 1995.

[26] J. W.-H. So And P. Waltman, A nonlinear boundary value problem arising from competition in the chemostat, Appl. Math. Comput., 32 (1989), pp. 169-183.

[27] J. Smoller, Shock Waves and Reaction-Diffusion Equations, Springer-Verlag, New York, 1983.

[28] D. Tilman, Resource Competition and Community Structure, Princeton University Press, Princeton, NJ, 1982. 
[29] P. Waltman, S. P. Hubbell, And S. B. Hsu, Theoretical and experimental investigation of microbial competition in continuous culture, in Modelling and Differential Equations, $\mathrm{T}$. Burton, ed., Marcel Dekker, New York, 1980.

[30] J. H. WU, Stability of steady-state solutions of the competition model in the chemostat, Systems Sci. Math. Sci., 7 (1994), pp. 256-260.

[31] J. H. Wu, Global bifurcation of coexistence state for the competition model in the chemostat, Nonlinear Anal., 39 (2000), pp. 817-835.

[32] J. H. Wu, AND G. S. K. WOLKOWICZ, A system of resource-based growth models with two resources in the unstirred chemostat, J. Differential Equations, 172 (2001), pp. 300-332.

[33] E. ZeIDler, Nonlinear Functional Analysis and its Application I: Fixed-Point Theorems, Springer-Verlag, New York, 1985. 